\title{
Porcelin and duplicated gallbladder associated with pancreatic cancer
}

\author{
Daniele Torres • Gaspare Parrinello • \\ Caterina Trapanese • \\ Mauro Cardillo · Giuseppe Licata
}

Received: 13 January 2011/Accepted: 18 March 2011/Published online: 31 March 2011

(c) SIMI 2011

Duplication of the gallbladder is a very rare clinical entity that is due to a congenital anomaly of the hepatobiliary system with a reported incidence of one per 4,000-5,000 persons, first described in a killed victim of the Emperor Augustus in 31 BC. It results from abnormalities in embryogenesis during the fifth and sixth weeks of gestation, and may be associated with some medical or surgical problems related to gallstones and cholecystitis [1] but, only rarely to gallbladder cancer [2]. Accurate pre-operative diagnosis of a double gallbladder is important to prevent possible surgical complications and repeated surgery when cholecystectomy is performed. Additionally, a porcelain gallbladder is another rare biliary condition characterized by the extensive calcification of the wall, sometimes associated with an obstructing cystic duct carcinoma, and is considered as a factor that may predispose to gallbladder cancer, but at a much lower rate than previously estimated [3]. In this brief report, we show a very

D. Torres $(\bowtie) \cdot$ G. Parrinello · C. Trapanese - M. Cardillo ·

G. Licata

Biomedical Department of Internal and Specialty Medicine

(Di.Bi.M.I.S.), A.O.U. "Policlinico Paolo Giaccone",

University of Palermo, Piazza delle Cliniche 2,

90127 Palermo, Italy

e-mail: daniele_torres@libero.it unusual combination of double biliary anomalies consisting of a duplicated and a porcelain gallbladder. The patient was a 75-year old woman admitted for 3 months history of asthenia, dyspepsia, anorexia, and weight loss $(3 \mathrm{~kg})$. Physical examination revealed a hard palpable mass in the right upper quadrant, and laboratory data demonstrated severe anemia ( $\mathrm{Hb} \quad 6.5 \mathrm{mg} / \mathrm{dl}$ ). Enhanced computed tomography (Fig. 1) to search for malignances, showed a double porcelain gallbladder separated by a shared medial wall, and united in the distal portion of the neck and infundibulum, with a unique main biliary duct. Also present were biliary sludge and an heteroplastic mass of the body and tail of the pancreas. In 1926, Boyden classified duplicate gallbladders as follows: bibbed incomplete gallbladder division with one cystic duct; complete gallbladder duplication with separate cystic ducts entering the common hepatic duct; and complete gallbladder duplication with a common cystic duct entering the common hepatic duct. Later, this classification was revised by Herlaftis et al. [4] on the basis of morphology and embryogenesis into two main groups and a third miscellaneous group. The split primordium group is characterized by the presence of a single cystic duct entering the common bile duct (Type 1). The accessory gallbladder group is characterized by two cystic ducts opening separately into the biliary tree (Type 2). Type 3 is a miscellaneous group that does differs from the foregoing two types (Fig. 2). In this report, we describe a rare type of duplicated gallbladder: the bilobed form, here accompanied by a porcelain gallbladder. Both anomalies were in association with a pancreatic cancer. While it is speculative to explain this association as an etiopathogenetic link, we cannot exclude that the probable bile-reflux into the pancreatic ducts, due to gallbladder anomalies, might be associated with the development of carcinoma, as previously documented in the literature [5]. 
Fig. 1 Computed tomography of the abdomen (a basal, b enhanced). The arrows indicate the duplicated porcelain gallbladder
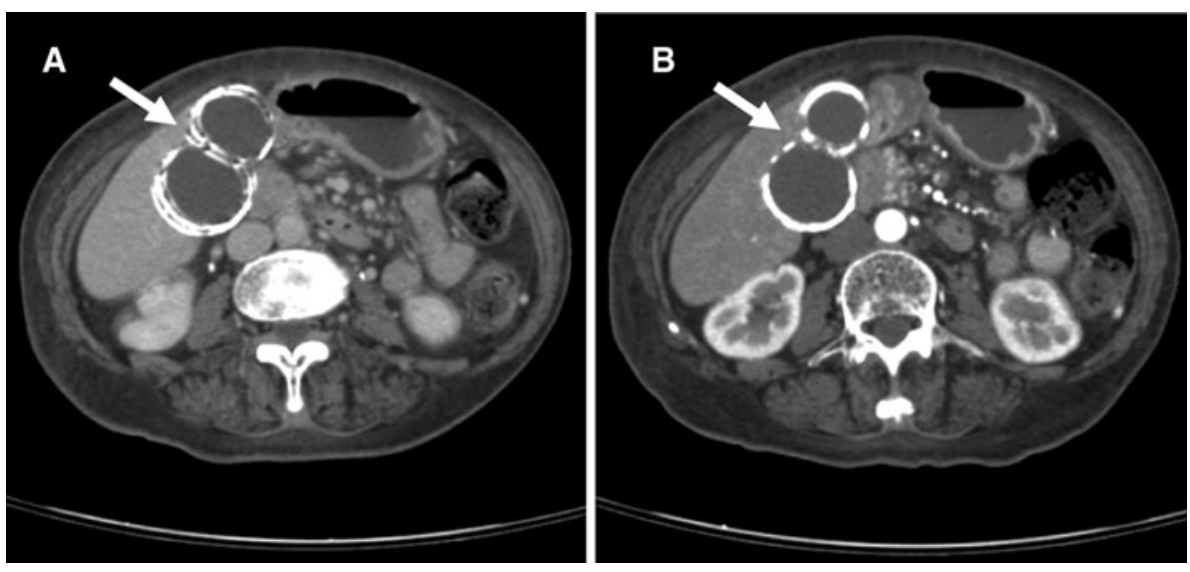

Type 1 : Split PrImordlum Group

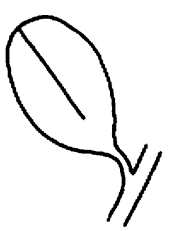

septate gallbladder

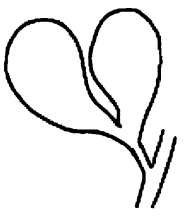

bilobed or $\mathrm{V}$ shaped

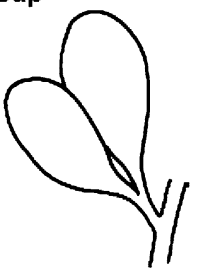

Y shaped
Type 2: Accessory Gallbladder Group

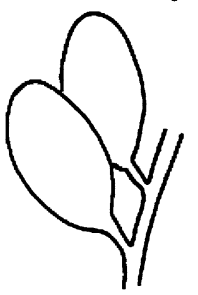

$\mathrm{H}$ or ductular

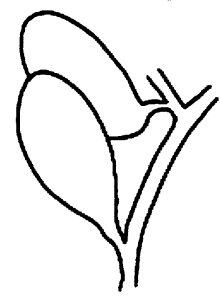

trabecular
Fig. 2 Classification of double gallbladder adopted from Herlaftis et al.

Conflict of interest None.

\section{References}

1. Hishinuma M, Isogai Y, Matsuura Y et al (2004) Double gallbladder. J Gastr Hepatol 19:233-235

2. Kin Y, Yamaguchi A, Isogai M, Hori A (1996) Duplication of the gallbladder with advanced adenocarcinoma: diagnostic value of MRI and angiography. Hepatogastroenterology 43(8):405-408

3. Stephen AE, Berger DL (2001) Carcinoma in the porcelain gallbladder: a relationship revisited. Surgery 129(6):699-703

4. Herlaftis N, Gray SW, Skandalakis JE (1977) Multiple gallbladders. Surg Gynecol Obstet 145:928-934

5. Adachi T, Tajima Y, Kuroki T, Mishima T, Kitasato A, Fukuda K, Fukuda K, Tsutsumi R, Kanematsu T (2006) Bile-reflux into the pancreatic ducts is associated with the development of intraductal papillary carcinoma in hamsters. J Surg Res 136(1):106-111 